\title{
New method to organ dose assessment in cone-beam computed tomography using ImPACT software
}

\author{
L. Hafezi ${ }^{1}$, D. Divband ${ }^{1}$, M.R. Deevband2 ${ }^{*}$ \\ ${ }^{1}$ Department of Oral and Maxillofacial Radiology, Faculty of Dentistry, Tehran Medical Sciences, Islamic Azad \\ University, Tehran Iran \\ ${ }^{2}$ Department of Medical Physics and Biomedical engineering, Faculty of Medicine, Shahid Beheshti University of \\ Medical Sciences and Health Services, Tehran, Iran
}

\section{Technical report}

\section{*Corresponding author: Mohammad R. Deevband, Ph.D., E-mail:}

mdeevband@sbmu.ac.ir

Revised: August 2020

Accepted: September 2020

Int. J. Radiat. Res., October 2021; 19(4): 1049-1053

DOI: $10.29242 /$ ijrr.19.4.1049

\begin{abstract}
Background: This study sought to assess the organ absorbed dose using ImPACT software and evaluate the effect of small and large fields of view (FOVs) for three cone-beam computed tomography (CBCT) devices on the organ absorbed dose. Materials and Methods: The weighted computed tomography dose index (CTDIw) is measured using a pencil ionization chamber which was incorporated in a polymethyl methacrylate (PMMA) phantom for three CBCT devices with small and large FOVs. The calculated CTDIw was entered into the ImPACT software. Then the organ absorbed dose of the thyroid gland, salivary glands, oral mucosa, skin, brain, and red bone marrow and the whole-body effective dose were calculated by the software.

Results: Irrespective of the type of CBCT device, the organ absorbed dose was obtained higher value in use of a larger FOV $(P<0.01)$. The mean organ absorbed dose in use of large and small FOVs was 0.13 and 0.08 for New Tom GiANO, 0.49 and 0.13 for Vista Vox $S$ and 0.69 and 0.38 for XMIND Trium, respectively. Salivary glands had the highest organ absorbed dose among all of the organs within the field. Larger FOVs yielded higher whole-body effective and organ absorbed doses compared with smaller FOVs. Conclusion: The results showed that using the ImPACT software to estimate the organ absorbed dose can serve as a suitable alternative to other costly and time-consuming methods available for this dose assessment in CBCT.
\end{abstract}

Keywords: Absorbed dose, organ absorbed dose, field of view, cone-beam computed tomography.

\section{INTRODUCTION}

Cone beam computed tomography (CBCT) is increasingly used for diagnosis of oral and dental conditions. Advanced computed tomography (CT) provides important data; but patient dose is several times higher than that of panoramic radiography ${ }^{(1)}$.Several methods have been employed to measure the organ absorbed dose in CBCT such as thermoluminescent dosimeters (TLD) which are costly and time-consuming method. Thus, this technique is not suitable for use as a routine applicable technique for quality control dosimetry (2-4). Different software programs developed for this purpose such as WinDose, ImPACT and CT-Expo. Dose calculations in mentioned software are performed according to reference phantoms and the dosimetry parameters are confirmed by the ICRP (5). Thus, the protocols used for estimation of the organ absorbed dose provide an estimate value, which are may differ from the actual organ absorbed dose. Considering the aforementioned problems in use of the direct method, the indirect method is now considered a valuable, reproducible and low-cost technique (3).

To the best of authors' knowledge, no previous study has evaluated CBCT dosimetry indirectly by use of computer software. 
Considering the gap of information in oral and maxillofacial imaging, this study aimed to use ImPACT software for estimation of absorbed dose that can serve as a suitable alternative to other costly and time-consuming methods available for this purpose.

\section{MATERIALS AND METHODS}

A polymethyl methacrylate (PMMA) cylindrical head phantom with $16 \mathrm{~cm}$ diameter and $15 \mathrm{~cm}$ length was used. This phantom had 5 holes parallel to the rotational axis of the phantom with $10 \mathrm{~mm}$ diameter. The central hole was located along the central axis of the phantom while the remaining four peripheral holes had $1 \mathrm{~cm}$ distance from the external surface of the phantom and located with $90^{\circ}$ angle relative to each other. For CTDI measurement, Barracuda dosimetry kit (Barracuda X-ray Analyzer, RTI Electronics, and Sweden) was used. This kit includes a pencil ionization chamber with serial number of 1673 which has an active length of $10 \mathrm{~cm}$. It was calibrated in a secondary standard dosimetry laboratory.

In order to obtain computed tomography dose index (CTDI) in (mGy), a pencil ionization chamber was placed in one of the five holes in the PMMA phantom and the rest of the holes were filled with acrylic plugs that came with the phantom. The head phantom was positioned in the CBCT device and underwent CBCT in standard mode. Then, the ionization chamber was placed in another hole and the above mentioned process was repeated. It should be noted that the exposure is repeated in five times for each position. Eventually, 5 CTDI values related to the five holes of the phantom were obtained and the weighted CTDIw was calculated using equation 1 .

$\mathrm{CTDI}_{\mathrm{w}}=1 / 3 \mathrm{CTDI}_{\mathrm{c}}+2 / 3 \mathrm{CTDI}_{\mathrm{p}}$

The $\mathrm{CTDI}_{\mathrm{c}}$ is the central value while the $\mathrm{CTDI}_{\mathrm{p}}$ is the mean four peripheral values (6).

The $\mathrm{CTDI}_{\mathrm{w}}$ was required to calculate the organ absorbed dose and whole-body effective dose in term of using ImPACT software version $2.3,2011$. The calculated $\mathrm{CTDI}_{\mathrm{w}}$ was entered into the ImPACT software along with the voltage $(\mathrm{kVp})$, gender, FOV and scanning duration. The organ absorbed dose for the thyroid gland, salivary glands, oral mucosa, skin, brain and red bone marrow and the whole-body effective dose were calculated using the software according to the ICRP103 guidelines (7). Table 1 lists the CBCT devices used in current study and their exposure settings. The measurements performed for small and large field of views (FOVs) in three CBCT devices which are now commonly used for diagnosis of oral and dental conditions namely XMIND Trium, Vista Vox S and NewTom GIANO in the year 2018 to 2019.

Table 1. The type of CBCT device, related techniques used in the study including related measured CTDIw in two FOVs.

\begin{tabular}{|c|c|c|c|c|c|}
\hline CBCT systems & $\begin{array}{c}\text { FOV } \\
\left(\mathbf{c m}^{\mathbf{2}} \mathbf{)}\right.\end{array}$ & mAs & Kvp & $\begin{array}{c}\text { CTDI } \\
\text { (mGy) }\end{array}$ & $\begin{array}{c}{ }_{\mathbf{n}} \mathbf{C T D I}_{\mathbf{W}} \\
\text { (mGy) }\end{array}$ \\
\hline \multirow{2}{*}{ NewTom GiANO } & $\mathbf{8 * 8}$ & 11.12 & 90 & 0.46 & 0.04 \\
\cline { 2 - 6 } & $\mathbf{8 * 5}$ & 11.12 & 90 & 0.48 & 0.04 \\
\hline \multirow{2}{*}{ VistaVox S } & $\mathbf{8 * 8}$ & 147.6 & 94 & 2.05 & 0.02 \\
\cline { 2 - 6 } & $\mathbf{5 * 5}$ & 121 & 98 & 0.71 & 0.01 \\
\hline \multirow{2}{*}{ XMIND Trium } & $\mathbf{6 * 6}$ & 48 & 110 & 3.34 & 0.07 \\
\cline { 2 - 6 } & $\mathbf{4} \mathbf{4}$ & 48 & 110 & 2.52 & 0.05 \\
\hline
\end{tabular}

\section{Statistical analysis}

SPSS (ver.16. SPSS Inc, USA, Chicago IL) and Microsoft Excel (Microsoft office professional plus 2013) soft wares were used for statistical analysis and analysis of variance (ANOVA) for comparison. A value of $\mathrm{p} \leq 0.05$ was assumed to indicate statistical significance. The obtained data were presented as the mean and standard deviation unless stated otherwise.

\section{RESULTS}

The calculated $\mathrm{CTDI}_{\mathrm{w}}$ for small and large FOVs for three CBCT devices presented in table 1. The results of organ absorbed dose for thyroid gland, salivary glands, oral mucosa, skin, brain, red bone marrow and whole body effective dose are presented in table 2. Irrespective of the type 
of CBCT device and size of FOV, the maximum organ absorbed dose (2.04 mGy) is related to salivary glands and it is due to the large FOV of XMIND Trium. The minimum organ absorbed dose $(0.25 \mathrm{mGy})$ is related to the small FOV of NewTom GiANO. The maximum whole-body effective dose $(0.06 \mathrm{mSv})$ is related to the use of large FOV of XMIND Trium device and it is followed by the use of large FOV of Vista Vox $S$ in an amount of 0.04 mGy and small FOV of XMIND Trium in an amount of 0.03 mGy. The other three protocols yielded values equal or smaller than $0.01 \mathrm{mGy}$. The mean organ absorbed dose (0.69 mGy) is calculated in large FOV for XMIND Trium, which is the highest one and followed by Vista Vox S and NewTom Giano in amounts of $0.49 \mathrm{mGy}$ and $0.13 \mathrm{mGy}$ respectively. The organ absorbed dose for XMIND Trium is 5.3 times the value in use of NewTom Giano. ANOVA test showed that this difference was significant $(\mathrm{P}<0.05)$.

Table 2. Organ absorbed dose (mGy) and the whole body effective dose (mSv) for different type of CBCT device and FOV size.

\begin{tabular}{|c|c|c|c|c|c|c|c|c|c|}
\hline \multicolumn{2}{|c|}{ Type of CBCT system } & $\begin{array}{c}\text { Thyroid } \\
\text { gland }\end{array}$ & $\begin{array}{c}\text { Salivary } \\
\text { glands }\end{array}$ & $\begin{array}{c}\text { Oral } \\
\text { mucosa }\end{array}$ & Skin & Brain & $\begin{array}{c}\text { Bone } \\
\text { marrow }\end{array}$ & $\begin{array}{c}\text { Mean and } \\
\text { tandard deviation }\end{array}$ & $\begin{array}{c}\text { whole body } \\
\text { Effective dose }\end{array}$ \\
\hline $\begin{array}{c}\text { NewTom } \\
\text { GiANO }\end{array}$ & $\mathbf{8} \mathbf{8} \mathbf{~ c m}^{\mathbf{2}}$ & 0.01 & 0.33 & 0.27 & 0.01 & 0.04 & 0.12 & $0.13 \pm 0.13$ & $<0.01$ \\
\hline \multirow{2}{*}{ Vista Vox S $\mathbf{2}$} & 0.01 & 0.25 & 0.19 & 0.01 & 0.01 & 0.00 & $0.08 \pm 0.11$ & $<0.01$ \\
\hline & $\mathbf{8} \mathbf{8} \mathbf{~ c m}^{\mathbf{2}}$ & 0.06 & 1.45 & 1.18 & 0.05 & 0.16 & 0.01 & $0.49 \pm 0.65$ & 0.04 \\
\hline $\begin{array}{c}\text { XMIND } \\
\text { Trium }\end{array}$ & $\mathbf{6} \mathbf{6} \mathbf{~ c m}^{\mathbf{2}}$ & 0.02 & 0.39 & 0.33 & 0.01 & 0.02 & 0.00 & $0.13 \pm 0.18$ & 0.01 \\
\cline { 2 - 11 } & $\mathbf{4} \mathbf{~ c m}^{\mathbf{2}}$ & 0.04 & 1.20 & 0.93 & 0.04 & 0.06 & 0.01 & $0.38 \pm 0.54$ & 0.06 \\
\hline
\end{tabular}

The post hoc test showed that the difference between NewTom Giano and Vista Vox S and also the difference between Vista Vox $S$ and XMIND Trium were not significant $(\mathrm{P}=0.6)$. The mean organ absorbed dose ( $0.39 \mathrm{mGy}$ ) in use of small FOV is related to the use of XMIND Trium and followed by Vista Vox S and NewTom Giano in amounts of 0.13 and 0.08 mGy respectively. The mean organ absorbed dose in use of XMIND Trium was 4.8 times the value in use of NewTom Giano. ANOVA test showed that the difference in this respect was significant $(\mathrm{P}<0.05)$. The post hoc test showed that the differences between NewTom Giano and Visa Vox S and also Vista Vox $S$ and XMIND Trium were not significant $(\mathrm{P}=0.6)$. Irrespective of the type of CBCT device, the mean organ absorbed dose in large FOVs was higher than that in small FOVs. According to t-test, this difference was statistically significant $(\mathrm{P}=0.01)$.

\section{DISCUSSION}

The maximum organ absorbed dose was found in salivary glands probably because of the location of salivary glands at the site of primary exposure ${ }^{8)}$. Other dosimetry studies have also confirmed this finding. The organ absorbed dose of the brain and red bone marrow was minimal probably due to the fact that they are only exposed to secondary beams. Also it seems that changing the size of FOV from small to large, would be increased in the organ absorbed dose which are located out of the primary radiation field. The maximum organ absorbed dose and the maximum whole-body effective dose were related to large FOV, obviously due to the exposure of a large body volume. The mean organ absorbed dose for both sizes of FOVs is belonged to XMIND Trium and it is higher than Vista Vox $S$ and NewTom GiANO, which is defined as a system with lowest mean organ absorbed dose. Low dose in NewTom GiANO is probably due to the lower exposure time and low amperage (3.6 s, $3.09 \mathrm{~mA}$ ) compared with two other systems. Among the three CBCT devices those are studied in current study, Vista Vox $\mathrm{S}$ was the only system that its $\mathrm{kVp}$ increased automatically by changing the size of FOV from large to small.

Ernest et al. (4) performed dosimetry for the 3D Accuitomo 170 CBCT system, using RT Humanoid phantom and TLD-100 chips and 
Monte Carlo simulations. Almost same kVp and mAs for NewTom GiANO device is considered in current study, which had the closest specification to those of used by Ernest et al. (4). Comparison of organ absorbed dose of the same organs for similar FOVs in the two studies revealed that the numerical values obtained by Ernest et al, were higher than current study. However, comparison of the organ absorbed dose from the highest to the lowest revealed that salivary glands had the highest value which followed by brain and thyroid gland and the findings in this respect were in line with the results of current study.

Sans Merce et al. evaluated the organ absorbed dose of the thyroid gland, parotid gland, and lens in CBCT using TLDs and a phantom equal to the size of an adult head (9). The entrance surface dose of phantom at the site of organs was calculated. Also, they used NewTom VGI CBCT system, which has a voltage similar to that of XMIND Trium in current study. The results of current study showed that the salivary glands received the maximum effective dose (0.94 mGy). In general, the doses obtained by Sans Merce et al. were much higher than the values in the present study, which may be due to the following reasons: (I) Sans Merce et al. calculated the entrance dose while in the present study, the organ absorbed dose were calculated, which are considerably lower than the entrance surface dose. (II) The size of FOV in the study by Sans Merce et al, was much larger than those which is used in present study.

Pauwels et al, evaluated the effect of FOV and angle of rotation on organ absorbed dose in CBCT. They showed that by decreasing the size of FOV particularly in the vertical dimension, a significant reduction occurred in the absorbed dose of patients, which was in agreement with finding of current study (8). Also, they demonstrated that in all FOVs, irrespective of the angle of rotation, the maximum organ absorbed dose is belonged to the salivary glands ( 0.58 to $5.74 \mathrm{mGy}$ ). The results presented by Pauwels et al. for the $8 \times 8 \mathrm{~cm}^{2} \mathrm{FOV}$ is compared with the results of current study. Both studies declare that the maximum organ absorbed dose is related to the salivary glands, which was in line with our findings, but the value of doses obtained by Pauwels et al. were much higher than those in current study, which may be due to higher amperage in their study (87.5).

Davies et al. measured organ absorbed dose of the jaws in CBCT using RANDO phantom and TLD-100H chips (10). The results (ranged from $0.032 \mathrm{mGy}$ to $0.078 \mathrm{mGy}$ ) were in line with the findings of current study (ranged from $0.03 \mathrm{mGy}$ to $0.06 \mathrm{mGy}$ ) and indicated that the organ absorbed dose had a direct correlation with the dimensions of the irradiated site.

These findings highlight the optimal efficacy of indirect dosimetry by use of a software program as an alternative for other dosimetry method. Nikneshan et al. (11), performed dosimetry for three CBCT devices with small and large FOVs using TLD and RANDO phantom. The organ absorbed dose and whole-body effective dose in smaller FOVs were higher, probably due to automatic increase in image resolution by changing the size of FOV from large to small (11).

This study had some limitations such as small number of CBCT devices evaluated in current study, wide geographical distribution of oral and maxillofacial radiology centers in the country. Similar studies using other software programs and Monte Carlo simulation for different type of CBCT devices are recommended.

\section{CONCLUSION}

The results show that using a software program such as ImPACT software to estimate the organ absorbed dose can serve as a suitable alternative to other costly and time-consuming methods available for the purpose of organ dosimetry. A software program was used for this purpose, which is more easily accessible and is easier to use compared with the commonly used techniques.

Conflicts of interest: Declared none. 


\section{REFERENCES}

1. Ludlow J, Davies-Ludlow L, Brooks S (2003) Dosimetry of two extra oral direct digital imaging devices: New Tom cone beam CT and Orthophos plus DS panoramic unit. Dentomaxillofacial Radiology, 32: 229-234.

2. Sykes JR, Lindsay R, Iball G, Thwaites DI (2014) Dosimetry of $\mathrm{CBCT}$ : methods, doses and clinical consequences. Conference Series; Journal of Physics, 1-13.

3. Dilek Cakmak E, Tuncel N, Sindir B, (2015) Assessment of organ dose by direct and indirect measurements for a wide bore X-ray computed tomography unit that used in radiotherapy. Int J Medical Physics, Clinical Engineering and Radiation Oncology, 4: 132-142.

4. Ernst M, Manser P, Dula K, Volken W, Stampanoni M, Fix MK ( 2017) TLD measurements and Monte Carlo calculations of head and neck organ and effective doses for cone beam computed tomography using 3D Accuitomo 170. Dentomaxillofacial radiology, 46: 1-12.

5. Karim MKA, Hashim S, Sabarudin A, Bradley DA, Bahruddin NA (2016) Evaluating organ dose and radiation risk of routine CT examinations in Johor, Malaysia. Sains Malaysiana, 45(4): 567-573.

6. Sadra H, Deevband M, Sardary D (2015) Estimated organ and effective dose from CT examinations using software impact at Shahid Beheshti University of Medical Sciences hospitals. The Quarterly journal of School of Medicine, Shahid Beheshti University of Medical Sciences, 39(1): 2629.

7. The 2007 Recommendations of the International Commission on Radiological Protection. ICRP Publication, 37(2-4): 1 $-332$.

8. Pauwels R, Beinsberger J, Collaert B, Theodorakou C, Rogers J, Walker A (2012) Effective dose range for dental cone beam computed tomography scanners. European Journal of Radiology, 81(2): 267-271.

9. Sans Merce M, Damet J, Becker M (2018) Comparative organ dose levels for dentomaxillofacial examinations performed with computed tomography, cone beam CT and panoramic radiographs. Radioprotection, 53(4): 287-291.

10. Davies J, Johnson B, Drage N, (2012) Effective doses from cone beam CT investigation of the jaws. Dentomaxillofacial Radiology, 41(1): 30-36.

11. Nikneshan S. Aghamiri MR, Moudi E, Bahemmat N, Hadian H (2016) Dosimetry of three cone beam computerized tomography scanners at different fields of view in terms of various head and neck organs. Iranian Journal of Radiology, 13(3): e34220. 
